\title{
BMJ Open Risk factors for predicting progression from normal cognition to mild cognitive impairment: protocol for a systematic review and meta-analysis of cohort studies
}

Jie Wang, ${ }^{\oplus 1}$ Lina Wang, ${ }^{1}$ Xianglian Zhou, ${ }^{1}$ Xiaohong Wen, ${ }^{2}$ Xueting Zhen ${ }^{1}$

To cite: Wang J, Wang $L$, Zhou X, et al. Risk factors for predicting progression from normal cognition to mild cognitive impairment: protocol for a systematic review and meta-analysis of cohort studies. BMJ Open 2019;9:e027313. doi:10.1136/ bmjopen-2018-027313

- Prepublication history and additional material for this paper are available online. To view these files, please visit the journal online (http://dx.doi. org/10.1136/bmjopen-2018027313)

Received 16 October 2018 Revised 15 March 2019 Accepted 15 May 2019

Check for updates

(C) Author(s) (or their employer(s)) 2019. Re-use permitted under CC BY-NC. No commercial re-use. See rights and permissions. Published by BMJ.

${ }^{1}$ School of Nursing, Huzhou University, Huzhou, Zhejiang, China

${ }^{2}$ Huzhou First People's Hospital, Huzhou, Zhejiang, China

Correspondence to

Dr Lina Wang;

aring2000@163.com

\section{ABSTRACT}

Introduction Mild cognitive impairment (MCl) often represents the earliest stage of Alzheimer's disease. There has been considerable research investigating specific risk factors regarding the progression from normal cognition to $\mathrm{MCl}$. However, different studies have come to different conclusions on the impact of particular risk factors.

Therefore, it is necessary to conduct a meta-analysis of the risk factors that predict cognitive disruption in individuals based on associations with $\mathrm{MCl}$.

Methods and analysis We will search seven electronic databases without time limit, including MEDLINE, EMBASE, The Cochrane Library, Web of Science, China National Knowledge Infrastructure, the Wan Fang Database and China Biology Medicine. Two researchers will independently screen for eligibility and perform data extraction. Data were extracted from cohort studies meeting the inclusive criteria according to the Newcastle Ottawa Scale (NOS) methods. A third member of the research team will be contacted when a consensus cannot be reached. Any disagreement will be settled by consensus. The NOS will be used to assess the quality of the studies. All analyses were performed using Stata V.15.1.

Ethics and dissemination We will report this review in accordance with the Preferred Reporting Items for Systematic Review and Meta-Analysis statement. We will disseminate our findings through a publication in a peerreviewed journal. This systematic review does not require ethical approval as no primary data are collected.

PROSPERO registration number CRD42018109099.

\section{BACKGROUND}

\section{Description of the condition}

The 2018 World Alzheimer Report reported that there are 50 million people with dementia in the world. This number is likely to increase to approximately 152 million by 2050 . Currently, the disease costs about a trillion US dollars a year and that cost is expected to double by 2030 . There is a new case every 3 seconds in the world, and there is no cure. Since 1998, approximately 100 drugs
Strengths and limitations of this study

- Previous studies of specific risk factors have only given approximations of the risks, which were affected greatly by the size, characteristics and other factors related to the population tested.

- This report will not only make use of the best available risk estimates but also explain the variability of these estimates under different genotypic and environmental backgrounds.

- Identifying numerous risk factors can help to guide development of new therapies and carry out early interventions to prevent the occurrence of mild cognitive impairment (MCl).

- The main result of this study will be to assess the risk factors for $\mathrm{MCl}$, without analysing risk factors for different types of $\mathrm{MCl}$ separately, which will have some limitations in explaining the correlation of risk factors with the specific MCl typing.

have been tested, and only four have been approved for use. These medications can help control some of the symptoms of dementia, but only for some people, and most people in the world do not have access to them. ${ }^{1}$

At present, clinical research and basic research have been turning to the preclinical stage, particularly the mild cognitive impairment (MCI) field, for guidance in the prevention and treatment of dementia. MCI is defined as cognitive impairment greater than expected for an individual's age and education level but that does not affect daily activities. ${ }^{2}$ The diagnosis of MCI can be based on the clinical consequences of the disease or the biological construct. Different guidelines have different diagnostic criteria for MCI, such as Diagnostic and Statistical Manual of Mental Disorders (DSM), National Institute on Aging-Alzheimer's Association (NIAAA) and National Alzheimer's Coordinating Center (NACC). ${ }^{3-5}$ The diagnostic criteria for 
MCI of 2018 NIA-AA Research Framework include the following: (1) Performance in the impaired/abnormal range on objective cognitive tests based on clinical judgement and/or on cognitive test performance. (2) In addition to evidence of cognitive impairment, evidence of decline in cognitive performance from baseline must also be present. May be characterised by cognitive presentations that are not primarily amnestic* (The 2018 NIA-AA Research Framework explained that the * at the end of the sentence means for MCI and dementia: Cognitive impairment may be characterized by presentations that are not primarily amnestic). (3) Performs daily life activities independently, but cognitive difficulty may result in detectable but mild functional impact on the more complex activities of daily life, either self-reported or corroborated by a study partner. ${ }^{4}$

Population-based studies estimate the prevalence of MCI ranging from $3 \%$ for subjects aged $\geq 60$ years to $15 \%$ for people aged $\geq 75$ years because the diagnostic criteria were used differently between studies. In a French population-based study of people aged $\geq 65$ years, the global incidence rate of MCI was 9.9/1000 person-years. In a Finnish population-based study, the global incidence rate of MCI was 25.94/1000 person-years. ${ }^{6}$

It has been reported that $10 \%-15 \%, 60.5 \%$ and $100 \%$ of MCI patients will develop full dementia within 1, 5 and 9.5 years, respectively, after the initial diagnosis of MCI. ${ }^{7}$ According to the Petersen diagnostic criteria, the main symptoms of MCI are memory loss and other cognitive impairments. ${ }^{89}$ A recent review described the prevalence of neuropsychiatric symptoms (such as depressive mood, irritability, emotional apathy, sleep disorders or agitation/aggression) in patients with MCI as $35 \%-85 \% .^{10}$ Therefore, the study of the conversion rate from normal cognition (NC) to MCI is important not only because of its contribution to understanding the overall progression towards Alzheimer's disease but also because MCI itself poses challenges for patients and families.

\section{Why is it important to do this review?}

The report noted that at least a third of the factors related to Alzheimer's disease can be intervened on or influenced. ${ }^{1}$ Kivipelto et al believes the figure could be closer to $50 \% .^{11}$

Cognitive impairment in MCI patients is the result of a combination of complex factors, including biological, psychological and social factors. Regarding the genetic contributions, the report observed that the combined effect of APOE genotype and depression was significantly greater than the sum of the independent effects of the two factors. ${ }^{12}$ The APOE $\varepsilon 4$ genotype can predict the general rate of decline in cognitive function. ${ }^{13}$

However, there is also evidence that APOE $\varepsilon 4$ risks for prevalent amnestic MCI (aMCI) vary depending on the definition of the objective neuropsychological impairment in MCI. ${ }^{14}$

In a large community-based study, atrial fibrillation (AF) was associated with a higher prevalence of MCI and dementia. ${ }^{15}$ However, when considering the correlation between $\mathrm{AF}$ and MCI, the baseline functional damage, as an important predictor of dementia, also needs to be taken into account and may help explain differences in findings between clinic-based studies and epidemiological studies. ${ }^{16}$

Additionally, if the observations were based on a small number of events, the associations between baseline mental state (such as disinhibition, hallucinations, delusions and euphoria) and the outcome of incident MCI should be considered preliminary. ${ }^{17}$ Yingjia Chen et al found that subjective cognitive complaints were a risk for future cognitive impairment, but she also thought that a large sample or further research was needed to extend the findings, such as that those who seek evaluation for their complaints are at particularly high risk. ${ }^{18}$

Although a considerable number of studies have been conducted on the specific risk factors contributing to the shift from NC to MCI, relatively little work has been done to predict cognitive disruption for individuals based on the factors' association with MCI.

To identify individuals in the NC population who are at the highest risk of MCI in the near future, specific risk factors and biomarkers that predict progress from NC to MCI need to be deeply understood. These people will then be a population targeted for cognitive interventions. Compared with a narrative synthesis, meta-analyses provide an accurate degree of consistency through quantitative variation. A quantitative synthesis will allow the development of MCI risk prediction models for future clinical studies by enumerating the different roles of published MCI risk factors. This protocol provides in-depth information on MCI, including research objectives and designs, search strategies, eligibility criteria, data extraction and synthesis, which are best suited for MCI researchers, clinicians and epidemiologists.

\section{OBJECTIVES}

The aim of this meta-analysis is to identify specific risk factors and biomarkers for the progression from $\mathrm{NC}$ to MCI.

\section{METHODS AND ANALYSIS}

\section{Study design and participants}

A literature search was expected to begin on July 25, 2018, with an expected completion date of December 31, 2018, providing time to determine the results of the study. The study will include all studies with well-defined MCI risk factors, either individually or in combination.

\section{Patient and public involvement}

No patients or the public will be involved in this study.

\section{Type of studies}

We will include cohort studies providing statistical data regarding risk factors associated with $\mathrm{MCI}$ incidence. The 
quality of the included studies will be examined independently by two reviewers using the Newcastle Ottawa Scale (NOS) to access the quality of each study, resolving disagreements by consensus, with the third reviewer contacted if consensus is not reached; low quality studies based on the NOS will be excluded. There is no restriction on language or publication status. The inclusion and exclusion criteria for the studies to be included in the sample are as follows.

Inclusion criteria will include the following: (1) the study used a cohort design; (2) the baseline population met the criteria for NC diagnosis; (3) the study assessed at least one risk factor for predicting progression from $\mathrm{NC}$ to MCI; (4) MCI was defined as the end point, the diagnosis criteria of MCI including DSM, Petersen's criteria, NIA-AA, NACC and others ${ }^{3-58919}$; (5) the publication had the longest follow-up period or the most comprehensive reporting of relevant data (the synthesis reports mainly indicated that the largest scale report has approximately one study or database) in situations where multiple articles came from a single study and (6) the study reported original data on relative risks (RRs), HRs or ORs and $95 \%$ CI or sufficient data to calculate the impact was provided.

Exclusion criteria are abstracts, review articles, commentaries, editorials, letters that reported no new data, hypothesis papers and meta-analyses.

\section{TYPES OF PARTICIPANTS}

\section{Inclusion criteria}

1. Participants of any age with NC will be considered.

2. Participants with a clearly confirmed diagnosis of NC.

\section{Exclusion criteria}

Participants' age or age ranges were not clearly indicated.

\section{Types of outcome measures}

The main result will be to assess the risk factors of MCI, including aMCI and non-amnestic MCI. We will report the ORs, RRs or HRs, and 95\% CI of relevant risk factors.

\section{Search methods for the identification of studies \\ Electronic searches}

According to key terms from previous literature reviews and Medical Subject Headings, we will search the following electronic bibliographic databases: MEDLINE, EMBASE, The Cochrane Library, Web of Science, China National Knowledge Infrastructure, the Wan Fang Database, and China Biology Medicine. To ensure saturation of the literature, we will further consult the list of references from the included studies or relevant reviews. Meanwhile, the bibliography of the included articles will be distributed to the review team.

The search strategy will include only terms relating to the risk factors. The search strategy we will use for the retrieval of reports of trials from the Cochrane Library can be seen in Appendix. The search strategy will be modified as necessary for other databases.

There will be no language restrictions. Studies published between January 1990 and the date of the searches will be sought. The searches will be re-run before the final analysis, and additional studies will be retrieved.

\section{Searching other resources}

Relevant cohort studies and reviews of additional studies will be hand-searched for more eligible studies. In addition, journals containing the most studies will be searched for recent publications ( $\leq 12$ months). Lastly, we will contact experts in the field of MCI and dementia to identify any unpublished or ongoing trials.

\section{Data collection and analysis}

Selection of studies

Two researchers will be reviewers and independently screen titles and abstracts of studies. Duplicates will be omitted using EndNote software (V.X7.8). ${ }^{20}$ Relevant studies will be selected according to the inclusion criteria. If necessary, reviewers will examine full-text reports to identify eligible studies. EndNote software will also be used to manage records. A third member of the research team will be contacted when a consensus cannot be reached. Any disagreement will be settled by consensus. An Excel spreadsheet has been developed to manage all review data. We will illustrate the selection process in a Preferred Reporting Items for Systematic Reviews and Meta-Analyses diagram. ${ }^{21}$

\section{Data extraction and management}

Search results will be uploaded into an EndNote database (X.7.8). Two reviewers will use a standardised and piloted extraction method to extract trial information independently, refer to the guidance document and resolve differences by discussion or the participation of a third reviewer.

Data will be double-extracted by two reviewers using a data extraction form developed in Excel for this review. Where possible, we will extract the following information related to the participant's characteristics and study design and contact the researchers in the case of missing data and any uncertainties. The detailed information will be extracted using a predefined data form as follows: general information (authors, title, year of publication, country of study), details of study design (aim, study type, risk factors related to MCI), study subjects (age, sex, sample size, number for analysis, other diseases) and outcomes (primary and secondary outcomes, follow-up strategies, method of outcome assessments, time points).

\section{Risk of bias of assessment}

The quality of the included studies will be examined independently by two reviewers, resolving disagreements by consensus; a third reviewer will be contacted when consensus is not reached. We will use the NOS to assess the quality of each research study, for which the score will 
range from 0 to 9 , and low-quality studies according to the NOS will be excluded.

Criteria for the NOS will include defined inclusion criteria, diagnostic criteria, statistical analysis, quality control, sample size and its representativeness. Studies with a score of $>5$ will be defined as high-quality studies. Studies with a score of $\leq 5$ will be defined as low-quality studies. $^{22}$

\section{Data synthesis and statistical analysis}

We will perform a multivariate analysis when studies provide more than one risk factor. Otherwise, a univariate analysis will be conducted. If three or more studies in a consistent manner report on a particular factor, we will combine them into a meta-analysis. Acquiescently, we will use a fixed effects model. Heterogeneity between studies will be assessed using the $\mathrm{I}^{2}$ statistic, and where statistically significant heterogeneity is found $\left(\mathrm{I}^{2}>50 \%\right.$, $\mathrm{p}<0.05$ ), a random effects model will be used to combine results. ${ }^{23}$ Prior to this, we will review the original literature and conduct a sensitivity analysis to examine the source of the heterogeneity. If data were not provided in a way that could be used in the meta-analysis or if only one or two studies were identified for a specific risk factor, the results of these studies will only be listed in the Discussion section. All analyses will be performed using Stata V.15.1.

\section{Dealing with missing data}

The lack of data in an individual trial may classify the study as a high risk of bias in the impact estimates. ${ }^{24}$ We will try to contact the original authors of the study to obtain the missing data. If the missing data cannot be obtained, an imputation method will be used. We will evaluate the important numerical data carefully.

\section{Assessment of heterogeneity}

Heterogeneity will be examined by calculating the $Q$ test and $\mathrm{I}^{2}$ statistic. If there is a high level of heterogeneity $\left(\mathrm{I}^{2} \geq 50 \%\right.$ or $\left.\mathrm{p}<0.1\right),{ }^{2526}$ we will try to explain the source of heterogeneity through a subgroup analysis.

\section{Sensitivity analysis and publication bias}

Sensitivity analysis will be performed by individually removing each study from the meta-analysis to examine whether the effect size was biased by the inclusion of any particular study. Selective outcome reporting bias will be detected using STATA V.15.1.

\section{Summary of evidence}

We will use the Grading of Recommendations Assessment Development and Evaluation approach to report the quality of evidence for all outcomes. Quality of evidence is rated as high, moderate, low and very low.

\section{Ethics and dissemination}

We will report this review in accordance with the PRISMA statement. We will disseminate our findings through a publication in a peer reviewed journal. Ethical approval is not required for the present systematic review as no primary data are collected. The protocol was registered with the International Prospective Register of Systematic Reviews (PROSPERO) on 19 September 2018. Any future amendments will be documented on the PROSPERO website.

\section{Amendments}

If we need to amend this protocol, the date of each amendment will be accompanied by a description of the change and the rationale.

Acknowledgements The authors thank Wang Xuegiang, member of the Spine Column of the International Cochrane evidence-based Center, for his assistance with the systematic review.

Contributors All authors contributed to the writing and redrafting of the manuscript. JW and XLZ designed the study. JW, XHW and XTZ wrote the protocol. XHW wrote the search strategy XLZ and XTZ conducted the literature searches, and JW wrote the draft of the manuscript. LNW provided critical revisions to the article, and all authors approved the final version of the article to be published.

Funding This work was supported by the National Natural Science Foundation (N0.71704053), Zhejiang Province Natural Science Foundation (NO. LQ17G030002), and China Scholarship Council.

Competing interests None declared.

Patient consent for publication Not required.

Provenance and peer review Not commissioned; externally peer reviewed.

Open access This is an open access article distributed in accordance with the Creative Commons Attribution Non Commercial (CC BY-NC 4.0) license, which permits others to distribute, remix, adapt, build upon this work non-commercially, and license their derivative works on different terms, provided the original work is properly cited, appropriate credit is given, any changes made indicated, and the use is non-commercial. See: http://creativecommons.org/licenses/by-nc/4.0/.

\section{REFERENCES}

1. Patterson C. World Alzheimer Report 2018:The state of the art of dementia research:3 New frontiers. Alzheimer's Disease International 2018.

2. Etgen T, Sander D, Bickel H, et al. Cognitive decline: the relevance of diabetes, hyperlipidaemia and hypertension. Br J Diabetes Vasc Dis 2010;10:115-22.

3. Association AP. Diagnostic and statistical manual of mental disorders: DSM-IV. Encyclopedia of the Neurological Sciences. , 2003:25, 4-8.

4. Jack CR, Bennett DA, Blennow K, et al. NIA-AA Research Framework: Toward a biological definition of Alzheimer's disease. Alzheimers Dement 2018;14:535-62.

5. Beekly DL, Ramos EM, van Belle G, et al. The National Alzheimer's Coordinating Center (NACC) Database: an Alzheimer disease database. Alzheimer Dis Assoc Disord 2004;18:270-7.

6. Panza F, D'Introno A, Colacicco AM, et al. Current epidemiology of mild cognitive impairment and other predementia syndromes. Am J Geriatr Psychiatry 2005;13:633-44.

7. Morris JC, Storandt M, Miller JP, et al. Mild cognitive impairment represents early-stage Alzheimer disease. Arch Neurol 2001;58:397-405.

8. Petersen RC, Smith GE, Waring SC, et al. Mild cognitive impairment: clinical characterization and outcome. Arch Neurol 1999;56:303-8.

9. Petersen RC. Mild cognitive impairment as a diagnostic entity. $J$ Intern Med 2004;256:183-94.

10. Monastero R, Mangialasche F, Camarda C, et al. A systematic review of neuropsychiatric symptoms in mild cognitive impairment. $J$ Alzheimers Dis 2009;18:11-30.

11. Kivipelto M, Solomon A, Ahtiluoto S, et al. The Finnish Geriatric Intervention Study to Prevent Cognitive Impairment and Disability (FINGER): study design and progress. Alzheimers Dement 2013;9:657-65.

12. Geda YE, Knopman DS, Mrazek DA, et al. Depression, apolipoprotein E genotype, and the incidence of mild cognitive impairment: a prospective cohort study. Arch Neurol 2006;63:435-40. 
13. Whitehair DC, Sherzai A, Emond J, et al. Influence of apolipoprotein E varepsilon4 on rates of cognitive and functional decline in mild cognitive impairment. Alzheimers Dement 2010;6:412-9.

14. Jefferson AL, Beiser AS, Seshadri S, et al. APOE and mild cognitive impairment: the Framingham Heart Study. Age Ageing 2015;44:307-11.

15. Alonso A, Knopman DS, Gottesman RF, et al. Correlates of Dementia and Mild Cognitive Impairment in Patients With Atrial Fibrillation: The Atherosclerosis Risk in Communities Neurocognitive Study (ARICNCS). J Am Heart Assoc 2017;6:e006014.

16. Farias ST, Mungas D, Reed BR, et al. Progression of mild cognitive impairment to dementia in clinic- vs community-based cohorts. Arch Neurol 2009;66:1151-7.

17. Geda YE, Roberts RO, Mielke MM, et al. Baseline neuropsychiatric symptoms and the risk of incident mild cognitive impairment: a population-based study. Am J Psychiatry 2014;171:572-81.

18. Chen $Y$, Denny KG, Harvey D, et al. Progression from normal cognition to mild cognitive impairment in a diverse clinic-based and community-based elderly cohort. Alzheimers Dement 2017;13:399-405.

19. Jak AJ, Bondi MW, Delano-Wood L, et al. Quantification of five neuropsychological approaches to defining mild cognitive impairment. Am J Geriatr Psychiatry 2009;17:368-75.

20. Song GM, Liu XL, Bian W, et al. Systematic review with network meta-analysis: comparative efficacy of different enteral immunonutrition formulas in patients underwent gastrectomy. Oncotarget 2017;8:23376-88.

21. Liberati A, Altman DG, Tetzlaff J, et al. The PRISMA statement for reporting systematic reviews and meta-analyses of studies that evaluate healthcare interventions: explanation and elaboration. BMJ 2009;339:b2700.

22. Zhang $\mathrm{M}$, Wang $\mathrm{N}$, Zhai Z, et al. Incidence and risk factors of chronic thromboembolic pulmonary hypertension after acute pulmonary embolism: a systematic review and meta-analysis of cohort studies. $J$ Thorac Dis 2018;10:4751-63.

23. Li JQ, Tan L, Wang HF, et al. Risk factors for predicting progression from mild cognitive impairment to Alzheimer's disease: a systematic review and meta-analysis of cohort studies. J Neurol Neurosurg Psychiatry 2016;87:476-84

24. Higgins J, Green SR. Cochrane handbook for systematic reviews of interventions.4.2.6. Naunyn-Schmiedebergs Archiv für experimentelle Pathologie und Pharmakologie 2009;5:S38.

25. Anthonappa RP, Ashley PF, Bonetti DL, et al. Non-pharmacological interventions for managing dental anxiety in children: John Wiley \& Sons, Ltd. 2017.

26. Boonstra A, van Dulmen-den Broeder E, Rovers MM, et al. Severe fatigue in childhood cancer survivors. Cochrane Database Syst Rev $2017 ; 85$. 